\title{
Formação de Membranas Planas Celulósicas por Espalhamento Duplo para os Processos de Nanofiltração e Osmose Inversa
}

\author{
Roberto B. de Carvalho, Cristiano P. Borges, Ronaldo Nobrega \\ Programa de Engenharia Química, COPPE, UFRJ
}

\begin{abstract}
Resumo: No presente trabalho é estudado o preparo de membranas anisotrópicas compostas, em uma única etapa, para os processos de NF e OI, através do espalhamento simultâneo de duas soluções poliméricas [acetato de celulose - AC/formamida/acetona (26,3/23,7/50 \% p/p) e AC/poli(vinil pirrolidona) - PVP/N,Ndimetilformamida - DMF (15/10/75 \% p/p)]. A precipitação das soluções, em água destilada, foi acompanhada por medidas de transmitância de luz. Estudou-se a influência das condições de preparo das membranas, na sua morfologia e nas suas propriedades de transporte (fluxo de permeado e retenção de solutos - Polietilenoglicol (PEG) para a NF e NaCl para a OI). Realizou-se também um tratamento térmico nas diferentes membranas produzidas. Os resultados mostraram que é possível obter membranas celulósicas com total aderência das diferentes camadas, a qual pode ser atribuída às condições de transferência de massa que retardam a precipitação na região próxima à interface das soluções espalhadas simultaneamente. Com relação aos testes de OI e NF, as membranas desenvolvidas apresentaram valores de fluxo permeado ( 7 - $465 \mathrm{~L} / \mathrm{h} \cdot \mathrm{m}^{2}$ ) dentro da faixa das membranas comerciais, retenção salina $(\mathrm{NaCl})$ entre $24-63 \%$ e retenção do PEG entre 53-82 \%, mostrando o potencial de aplicação destas membranas.
\end{abstract}

Palavras-chave: Acetato de celulose, osmose inversa, nanofiltração, processo de inversão de fases, espalhamento duplo.

\section{Membranes for Nanofiltration and Reverse Osmosis Prepared by Simultaneous Casting of Two Polymer Solutions.}

Abstract: The purpose of this work is to investigate the preparation of composite anisotropic membranes in a single stage, through the technique of simultaneous casting of two polymer solutions (cellulose acetate (CA)/ acetone/formamide $(26.3 / 50 / 23.7 \% \mathrm{w} / \mathrm{w})$ and CA/polyvinylpyrrolidone/dimethyl formamide $(15 / 10 / 75 \%$ $\mathrm{w} / \mathrm{w})$ ). The precipitation of the solutions was followed using light transmission experiments. The effect of the exposition period precipitation bath on the membrane properties is also reported. All the membranes were characterized by scanning electron microscopy and by permeation experiments. The RO and NF experiments are carried out using $5,000 \mu \mathrm{g} / \mathrm{g}(\mathrm{ppm})$ of $\mathrm{NaCl}$ aqueous solution and $5 \mathrm{~g} / \mathrm{L}$ of polyethyleneglycol (PEG), 6 $\mathrm{KDa}$, aqueous solution, respectively. Moreover, to reduce defects in the upper layer, the membranes were immersed in a water bath at $90^{\circ} \mathrm{C}$ for 60 minutes. The membrane morphology analysis indicates that is possible to obtain complete adhesion of the two layers. This result is related to the mass transfer conditions, which should delay the precipitation in the regions near the polymer solutions interface. Regarding the performance of the membranes in RO and NF experiments, the permeate fluxes are comparable to those observed with commercial membranes $\left(7-465 \mathrm{~L} / \mathrm{h} . \mathrm{m}^{2}\right)$. The solute retention varied in the range of 24 to $63 \%$ for $\mathrm{NaCl}$, and in the range of 53 to $82 \%$ for PEG. These results point out the potential of technical scale application of the double layer membranes.

Keywords: Cellulose acetate, reverse osmosis, nanofiltration, phases inversion, simultaneous casting.

Autor para correspondência: Roberto B. de Carvalho, Programa de Engenharia Química (PEQ), COPPE, UFRJ, Centro de Tecnologia, CEP: 21945-970, Cidade Universitária, Rio de Janeiro, RJ. E-mail: bentes@peq.coppe.ufrj.br 


\section{Introdução}

Os processos de separação por membranas (PSM) podem ser empregados em um vasto número de aplicações em diferentes áreas, tais como: médica, biológica e farmacêutica, e em indústrias químicas e de alimen$\operatorname{tos}^{[1-3]}$. A osmose inversa (OI) é um PSM amplamente utilizado na dessalinização, no reuso e no tratamento de águas, mostrando-se como uma alternativa bastante viável para a resolução do problema da escassez mundial de água potável, que é uma preocupação crescente neste novo milênio. Embora a OI seja um processo já empregado em escala industrial, existem ainda algumas limitações que podem ser superadas com o desenvolvimento de novas gerações de membranas. A nanofiltração (NF), por sua vez, é um PSM capaz de promover separações de moléculas numa ampla faixa de peso molecular, podendo ser empregado na indústria de química fina (separação de moléculas orgânicas), e também na recuperação de moléculas com altos valores agregados (fármacos, enzimas e biocatalizadores). A classificação deste processo (NF) ainda é recente, sendo que o seu mercado ainda está em desenvolvimento e o mesmo caracteriza-se pela ausência de membranas de alta eficiência.

O desenvolvimento de membranas anisotrópicas, pelos pesquisadores LOEB e SOURIRAJAN ${ }^{[4]}$, utilizando o processo de inversão de fases, é considerado o marco principal da elevação dos PSM à escala industrial. Estas membranas consistem de uma camada superior fina (denominada "pele"), densa ou com poros muito pequenos, responsável pela seletividade, suportada sobre um suporte poroso, que proporciona resistência mecânica à "pele" e oferece pouca resistência ao transporte. Se a pele e o suporte forem do mesmo material a membrana é dita anisotrópica integral, caso contrário, denomina-se anisotrópica composta.

Um dos maiores desafios científicos encontrados no desenvolvimento de membranas com esta estrutura morfológica é a dificuldade de se obter uma pele ultrafina que seja isenta de defeitos ${ }^{[3,5]}$. Nos últimos anos, este problema é solucionado utilizando-se membranas porosas ou anisotrópicas como suporte, sobre as quais são realizadas sucessivas etapas de cobrimento com o intuito de formar a pele ou fechar os defeitos da mesma, obtendo assim membranas anisotrópicas compostas em multietapas. $\mathrm{O}$ contato de uma solução diluída sobre a superfície do suporte, polimerização interfacial e polimerização iniciada por plasma, são algumas das técnicas de cobrimento freqüentemente utilizadas ${ }^{[6,7]}$.

No presente trabalho emprega-se uma nova técnica que possibilita o preparo de membranas anisotrópicas compostas em uma única etapa. Com esta finalidade, membranas planas de dupla camada são produzidas através do espalhamento simultâneo de duas soluções poliméricas, utilizando uma faca de espalhamento duplo, formando a pele seletiva e o suporte poroso em uma única etapa. Futuramente o desenvolvimento de membranas duplas do tipo fibraoca, através da extrusão simultânea das duas soluções poliméricas utilizando-se uma extrusora tripla, também será estudado.

Comparando a técnica de espalhamento duplo com as técnicas convencionais de preparação de membranas anisotrópicas compostas, pode-se destacar as seguintes vantagens: a redução no número de etapas do processo, o aumento da porosidade do suporte poroso na interface com a pele, a adaptação da espessura das camadas através das condições de preparo da membrana, a possibilidade de utilização de um maior número de materiais nas duas soluções poliméricas e maiores possibilidades de otimização da pele e da subcamada. $\mathrm{O}$ fator limitante desta nova técnica é a aderência entre as duas camadas, mas também é possível a formação de uma pele entre as camadas, o que afetaria as propriedades de transporte da membrana.

Nos primeiros trabalhos sobre membranas de dupla camada, publicados por WEIGEL et al. ${ }^{\text {op.cit.[6] }}$, as duas soluções poliméricas eram espalhadas e depois imersas em um banho de precipitação, formando membranas com diferentes características nas duas camadas. Os polímeros utilizados foram poliacrilonitrila, acetato de celulose e poliuretano. Apesar desta nova técnica ser bastante atrativa, não se encontram na literatura trabalhos referentes à utilização do espalhamento duplo para obtenção de membranas de NF e OI, sendo a mesma mencionada somente na preparação de membranas para os processos de diálise, separação de gases e ultrafiltração ${ }^{[5,6]}$.

O objetivo deste trabalho foi o desenvolvimento de membranas anisotrópicas com pele ultrafina, através da técnica de espalhamento duplo, empregando o acetato de celulose como polímero base. Estudou-se o comportamento de algumas variáveis envolvidas no preparo das membranas planas duplas, objetivando melhor compreensão dos fenômenos envolvidos na 
formação das mesmas, e também correlacionar estas variáveis com a sua morfologia e com as suas propriedades de transporte, visando obter membranas adequadas aos processos de nanofiltração e osmose inversa.

\section{Formação da Membrana}

As membranas são formadas pelo processo de inversão de fases, o mesmo é amplamente utilizado no preparo de membranas comercias e permite a obtenção de membranas com diferentes tipos de morfologia e propriedades de transporte. Este processo caracteriza-se por transformações nas soluções poliméricas, devidas a alterações na sua concentração ou temperatura, levando a sua instabilidade termodinâmica, promovendo a separação destas em pelo menos duas fases ${ }^{[4]}$.

A técnica de espalhamento duplo permite uma combinação sinergética de diferentes concepções para a indução da instabilidade termodinâmica das soluções poliméricas. Neste trabalho, utilizou-se a evaporação controlada de solvente, para formação da pele, seguida da inversão de fases por imersão-precipitação, para formação do suporte poroso (Figura 1).

$\mathrm{Na}$ evaporação controlada utiliza-se uma solução constituída de um polímero (P), um solvente (S) volátil - acetona e um não-solvente (NS) menos volátil - formamida. A evaporação do solvente provoca aumento da concentração de polímero na solução até que a precipitação ocorra, devido à presença do nãosolvente. A alta concentração de polímero pode levar à formação da pele devido a efeitos viscosos promo-

Formação da pele

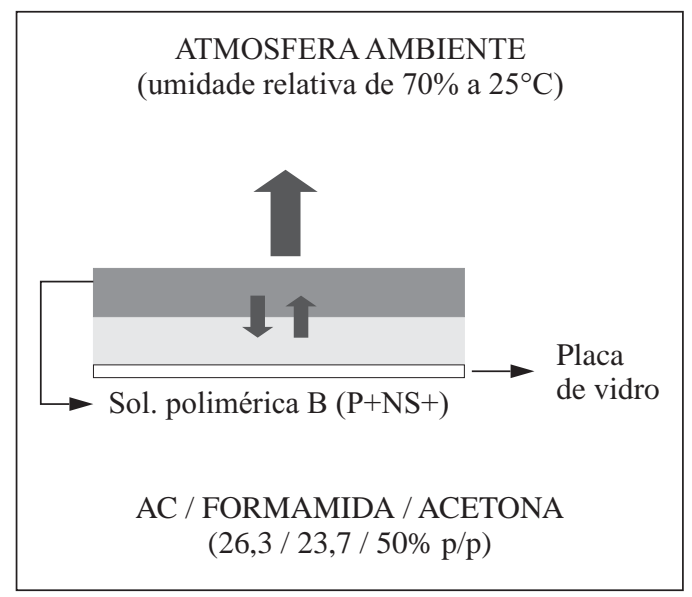

Evaporação Controlada de Solvente vidos pelos fenômenos de cristalização, gelificação ou vitrificação.

$\mathrm{Na}$ inversão de fases por imersão-precipitação, a solução é espalhada sobre uma placa de vidro e imersa num banho de não-solvente para o polímero. A transferência de massa entre a solução polimérica e o banho promove a separação de fases líquido-líquido, dando origem a uma fase rica e a outra pobre em polímero. $\mathrm{O}$ aumento da concentração da fase rica em polímero leva à formação do suporte poroso. Esta concepção é muito flexível e permite obter suportes com diferentes morfologias, dependendo da escolha do solvente e do não solvente ao polímero, da utilização de aditivo (AD) na solução polimérica, como também da composição do banho de precipitação. Utilizou-se poli(vinil pirrolidona) (PVP) como aditivo na solução polimérica formadora do suporte poroso. O PVP melhora a interconectividade dos poros das subcamadas, oferecendo pouca resistência ao transporte, o que aumenta a permeabilidade da membrana.

A transferência de massa entre as duas soluções poliméricas afeta a morfologia das membranas duplas produzidas, e é fator determinante na aderência das duas camadas e na compreensão dos fenômenos envolvidos na síntese das mesmas.

A técnica de espalhamento duplo tem sido estudada no Laboratório de Processos de Separação Através de Membranas (PEQ/COPPE) nos últimos cinco anos, com bons resultados no preparo de membranas para os processos de separação de gases ${ }^{[5]}$ e de osmose inversa/nanofiltração ${ }^{[8]}$.

Formação do suporte poroso

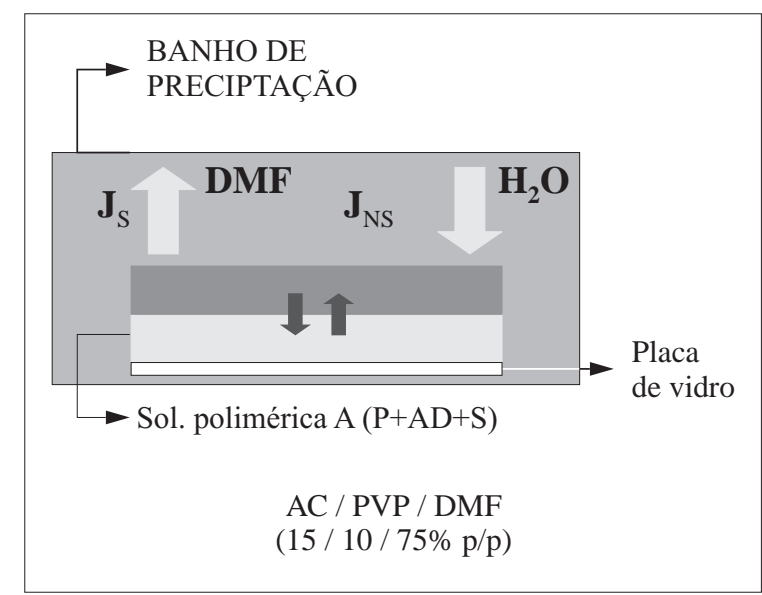

Inversão de Fases por

Precipitação - Imersão

Figura 1. Concepções utilizadas para induzir a instabilidade das diferentes soluções. 


\section{Experimental}

\section{Materiais}

O acetato de celulose - AC (tipo S4R) foi fornecido pela Rhodia Brasil Ltda., em forma de grãos e usado após secagem em estufa a $60^{\circ} \mathrm{C}$ por no mínimo de 24 horas. O aditivo polivinilpirrolidona - PVP (tipo K90), adquirido da Fluka Chemika Co., foi seco sob as mesmas condições. A acetona, o N,N-dimetilformamida DMF, a formamida, o n-hexano, todos com grau de pureza analítico, e o etanol (95\%), foram adquiridos da Vetec Química Fina Ltda. e usados sem tratamento prévio. Utilizou-se água destilada como banho de precipitação. As membranas produzidas foram armazenadas em solução aquosa de azida de sódio (Vetec, 99\%). As características seletivas das membranas foram medidas através de testes de permeação com soluções aquosas dos seguintes solutos: polietilenoglicol (PEG) 6.000 Dalton (Fluka Chemika Co.), testes de NF, e $\mathrm{NaCl}$ (Berzog, 99,5\%), testes de OI.

\section{Preparo das Membranas}

As soluções poliméricas foram preparadas adicionando-se os componentes, em um Erlenmeyer, o qual era fechado e vedado com Parafilm ${ }^{\circledR}$, e colocado sob agitação magnética constante. A composição da $S o$ lução $A$, que deu origem à camada inferior (suporte poroso), foi: AC/PVP/DMF (15/10/75\%p/p). Já a Solução $B$, que foi responsável pela formação da ca- mada superior (pele), continha: $26,3 \% \mathrm{p} / \mathrm{p}$ de $\mathrm{AC}$, $23,7 \% \mathrm{p} / \mathrm{p}$ de formamida e $50 \% \mathrm{p} / \mathrm{p}$ de acetona.

No preparo das membranas de camada dupla utilizou-se uma faca de espalhamento especial, com dois bastões de vidro conectados paralelamente entre si, o que permitia o espalhamento duplo das duas soluções poliméricas. A placa de vidro utilizada possuía uma diferença de nível, de forma que a solução B fosse colocada num nível superior. Um dos bastões espalhava a solução A, enquanto, o outro bastão espalhava simultaneamente, sobre esta, a solução B. Após espalhamento o filme polimérico era então exposto à atmosfera ambiente (umidade relativa de $70 \%$ a $\left.25^{\circ} \mathrm{C}\right)$ por diferentes tempos $(10 \mathrm{~s}, 30 \mathrm{~s}$ e $60 \mathrm{~s}$ e 30 min) e, em seguida, imerso no banho de precipitação de água destilada. Depois da precipitação, as membranas permaneciam em banho de água a $60^{\circ} \mathrm{C}$ por 8 $\mathrm{h}$, para retirada de solvente residual da matriz polimérica. A secagem das membranas foi realizada através da técnica de troca de solvente (primeiro etanol por três horas, e então por mais três horas em n-hexano). As etapas envolvidas no preparo de membranas planas de camada dupla são esquematizadas na Figura 2.

\section{Tratamento Térmico}

Com o objetivo de minimizar os defeitos da superfície superior da membrana, visando o aumento da seletividade da mesma, realizou-se o tratamento térmico das diferentes membranas de dupla camada
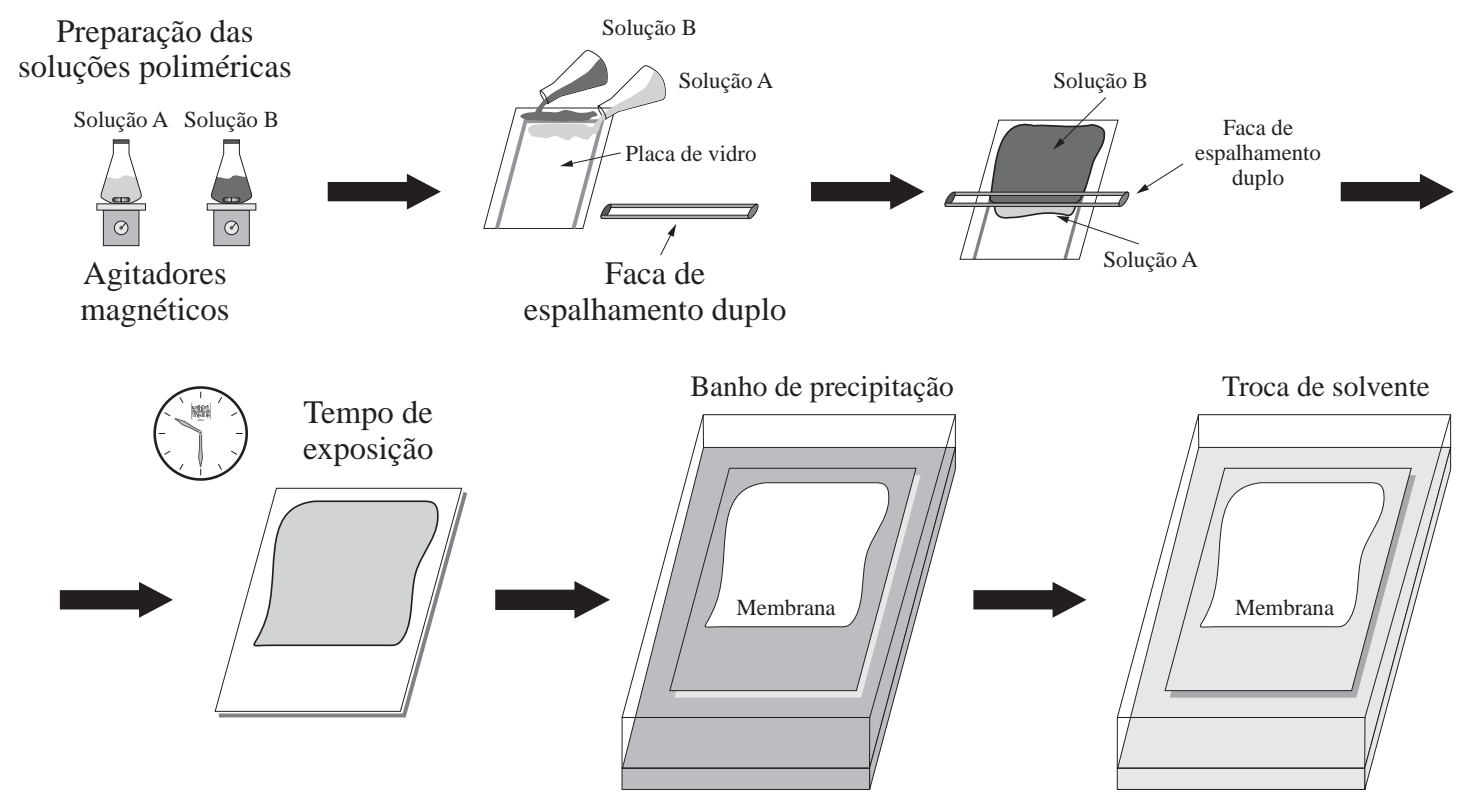

Figura 2. Etapas envolvidas no preparo de membranas por espalhamento duplo. 
produzidas. Este tratamento consistia basicamente na imersão das membranas, suportadas e protegidas por duas placas de vidro, num banho isotérmico $\left(90^{\circ} \mathrm{C}\right)$ de água microfiltrada durante 1 hora. Cabe mencionar que a temperatura do banho isotérmico e o tempo de duração deste tratamento foram escolhidos com base em trabalhos anteriores ${ }^{[9]}$.

Neste tratamento, devido ao aumento da mobilidade segmental do polímero, ocasionado pelo aumento da energia térmica, há um aumento do número de choques entre as moléculas e da possibilidade de formar ligações intermoleculares que, irreversivelmente, reduzem o volume livre da matriz polimérica, provocando uma redução no tamanho dos poros superficiais por contração capilar, verificada através de um aumento na seletividade e de uma diminuição na permeabilidade da membrana tratada ${ }^{[10]}$.

\section{Caracterização das Membranas}

\section{Morfologia}

Todas as membranas produzidas foram caracterizadas quanto à sua morfologia em um microscópio eletrônico de varredura (MEV), Jeol, modelo JSM 5300. As fotomicrografias das membranas eram processadas utilizando-se um analisador de imagens (Zeiss). Antes de analisadas no MEV, as amostras das membranas secas eram fraturadas em nitrogênio líquido (para evitar deformação na seção transversal), colocadas em um suporte e cobertas com uma espessura de aproximadamente $300 \AA$ de ouro, num metalizador da Jeol, modelo JFC 1500.

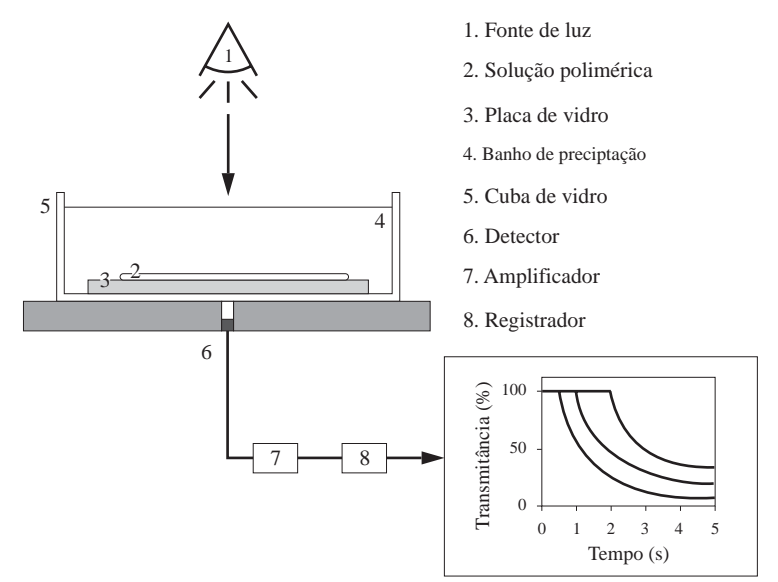

Figura 3. Representação esquemática do equipamento utilizado para determinar a velocidade de precipitação das soluções poliméricas.

\section{Velocidade de precipitação}

A velocidade de precipitação das duas soluções poliméricas, espalhadas de modo simultâneo, foi acompanhada através da queda de transmitância de luz com o tempo, utilizando-se o equipamento apresentado na Figura 3. Este equipamento consiste de uma fonte de luz (lâmpada $60 \mathrm{~W}$ ) e um sensor fotoelétrico conectado a um computador, onde ocorria a aquisição de dados.

\section{Propriedades de transporte}

A representação esquemática da unidade de nanofiltração e de osmose inversa, utilizada para caracterização das propriedades de transporte (fluxo de permeado e rejeição de solutos) das diferentes membranas de camada dupla, é apresentada na Figura 4. A área útil de permeação era de $20,43 \mathrm{~cm}^{2}$. O volume morto do sistema era de $280 \mathrm{~mL}$.

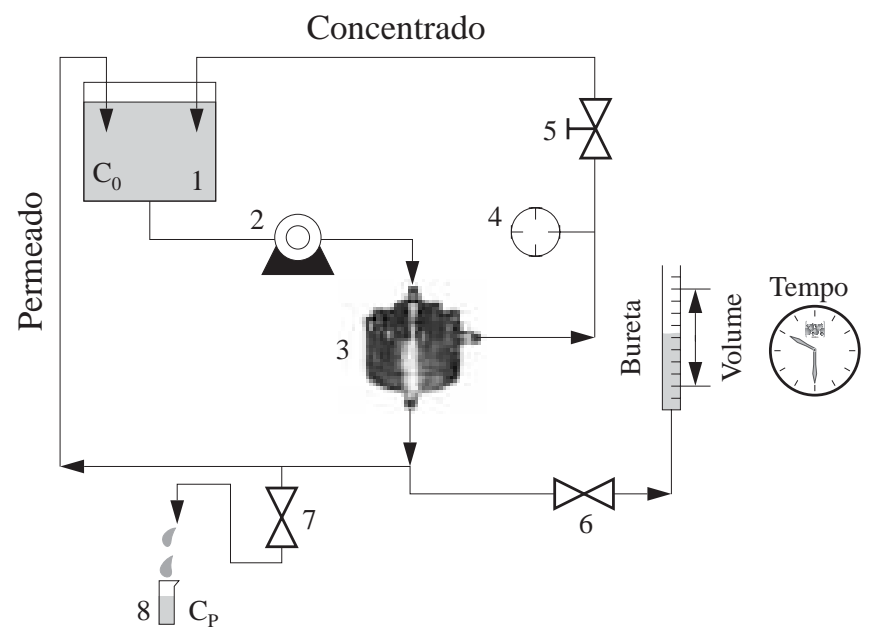

1. Tanque de alimentação

2. Bomba

3. Célula de permeação

4. Medidor de pressão

5. Válvula reguladora de pressão

6. Válvula para o medidor de vazão

7. Válvula para a coleta de permeado

8. Recipiente para a amostragem do permeado

Figura 4. Representação esquemática da unidade de nanofiltração e de osmose inversa empregada na compactação e caracterização das membranas. 
Inicialmente as membranas eram submetidas a testes de compactação, visando obter fluxos de permeado, $\left[\mathrm{L} /\left(\mathrm{m}^{2} . \mathrm{h}\right)\right]$, constante com o tempo. Nesta etapa, o sistema foi operado à temperatura ambiente $\left(\mathrm{T}=25^{\circ} \mathrm{C}\right) \mathrm{e}$ pressão predeterminada $(\mathrm{P}=28$ bar), sendo o mesmo alimentado com água destilada e microfiltrada (diâmetro de poros $0,45 \mu \mathrm{m}$ ) com pequenas quantidades de azida de sódio (evitar desenvolvimento de microorganismos na superfície das membranas), com um volume de aproximadamente 2 litros. Determinava-se o fluxo de água permeada $\left(\mathrm{J}_{\mathrm{P}}\right)$. Em seguida realizava-se a etapa de caracterização das membranas, operando o sistema nas mesmas condições operacionais de temperatura e pressão da etapa de compactação.

Novas membranas, preparadas sob as mesmas condições de síntese das caracterizadas anteriormente, eram submetidas ao tratamento térmico. Estas membranas tratadas também eram compactadas e caracterizadas quanto às suas propriedades de transporte.

O sistema era alimentado com uma solução aquosa microfiltrada de $\mathrm{NaCl}$ (5000 ppm) e/ou com uma solução aquosa microfiltrada de polietilenoglicol 6000 Dalton $(5 \mathrm{~g} / \mathrm{L})$, sendo que as propriedades de transporte empregadas para caracterização das diferentes membranas produzidas, eram: o fluxo de permeado, e a rejeição aos mesmos solutos [Equação (1)].

$$
\mathrm{R}(\%)=\left[\left(\mathrm{C}_{0}-\mathrm{C}_{\mathrm{p}}\right) / \mathrm{C}_{\mathrm{o}}\right] \mathrm{x} 100
$$

onde;

$\mathrm{C}_{\mathrm{o}}=$ concentração de soluto na alimentação (ppm ou g/L) $\mathrm{C}_{\mathrm{P}}=$ concentração de soluto no permeado $(\mathrm{ppm}$ ou $\mathrm{g} / \mathrm{L})$

A análise do teor salino do permeado foi feita por medidas de condutividade elétrica (condutivímetro modelo CD-21, Digimed). Nos testes de rejeição ao PEG, medidas de índice de refração (Shimadzu, modelo RID-10A) foram utilizadas para calcular o teor do soluto no permeado. As concentrações de permeado, utilizadas no cálculo das rejeições ao $\mathrm{NaCl}$ e ao PEG, foram determinadas através de curvas de calibração.

\section{Resultados e Discussão}

\section{Velocidade de Precipitação e Morfologia das Membranas}

A Figura 5 mostra o efeito do tempo de exposição do filme polimérico ao ambiente sobre a velocidade de precipitação das soluções A (solução do suporte poroso) e B (solução da pele), espalhadas simultanea-

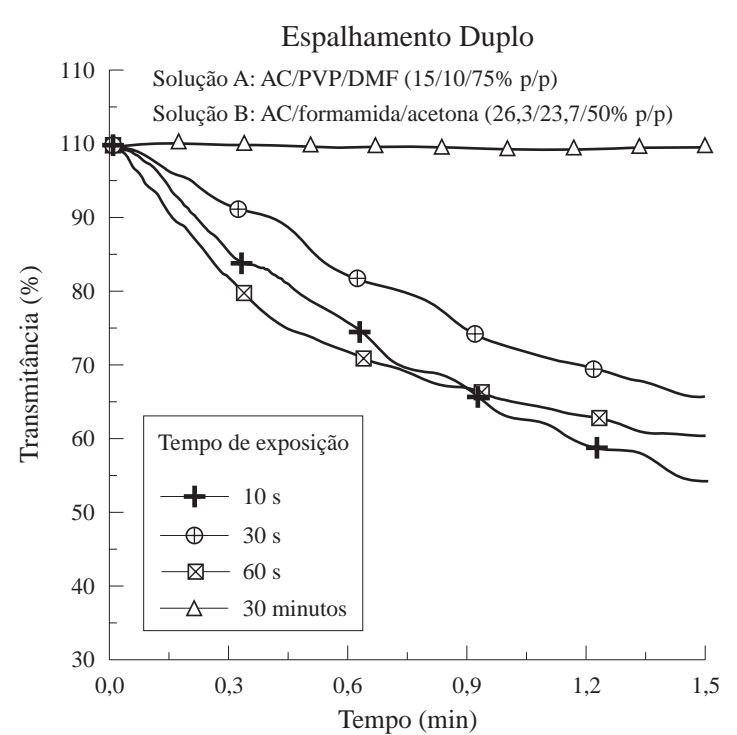

Figura 5. Medidas de transmitância de luz, variando o tempo de exposição do filme polimérico ao ambiente, para as soluções processadas por espalhamento duplo.

mente. Nos testes em que o filme polimérico foi exposto ao ambiente por 10,30 e 60 s, a precipitação começava a ser acompanhada após a imersão da placa de vidro no banho de precipitação. Já nas medidas com exposição prolongada ao ambiente (30 minutos), acompanhava-se a transmitância de luz durante o tempo de exposição, ou seja, sem banho de precipitação.

Pode-se perceber na Figura 5, que as quedas da transmitância de luz com o tempo, para os filmes expostos por $10 \mathrm{~s}$ e $60 \mathrm{~s}$ ao ambiente (umidade relativa de $70 \%$ a $25^{\circ} \mathrm{C}$ ), são mais rápida que a do filme exposto por 30s. Nota-se aqui uma alteração do comportamento da velocidade de precipitação com o aumento do tempo de exposição, verificada devido à aceleração da precipitação quando o filme polimérico foi exposto por 60s à atmosfera ambiente. Esta mudança de comportamento pode ser atribuída a dois efeitos antagônicos que podem ocorrer com o aumento do tempo de exposição. $\mathrm{O}$ aumento deste tempo aproxima o sistema da região de separação de fases atuando no sentido de acelerar a precipitação. Esta aproximação pode ocorrer devido, principalmente, a evaporação da acetona contida na solução da pele, que provoca o aumento da concentração do não solvente para o polímero (formamida), ou ainda pela absorção de uma maior quantidade de água (não solvente) durante o tempo de exposição. Por outro lado, quando o tempo de exposição aumenta, podese esperar também uma maior concentração de polímero na interface entre o banho de precipitação e 
o filme polimérico, aumentando a resistência à transferência de massa atuando no sentido de retardar a precipitação da subcamada abaixo da interface.

A transferência de massa entre as duas soluções poliméricas pode atuar tanto no sentido de atrasar a precipitação, como também pode contribuir para aproximar as soluções da região de separação de fases.

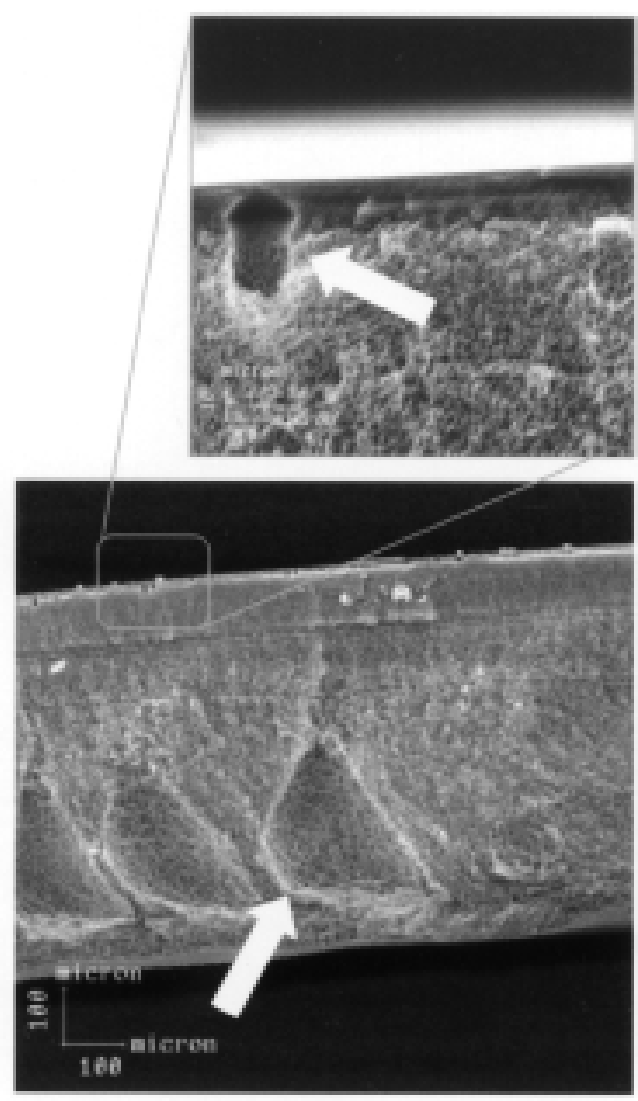

$10 \mathrm{~s}$
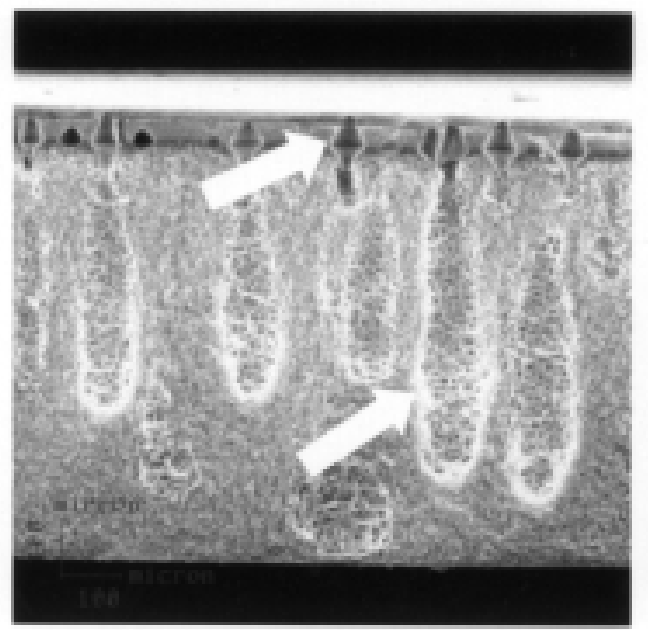

$60 \mathrm{~s}$
Por exemplo, supondo a difusão de DMF da solução A em direção a solução $B$, além de estabilizar a camada da pele (atrasar a precipitação), a saída de DMF da solução provocaria uma aceleração da precipitação da subcamada porosa.

Para o filme exposto ao ambiente por 30 minutos, não se observa queda na transmitância de luz durante

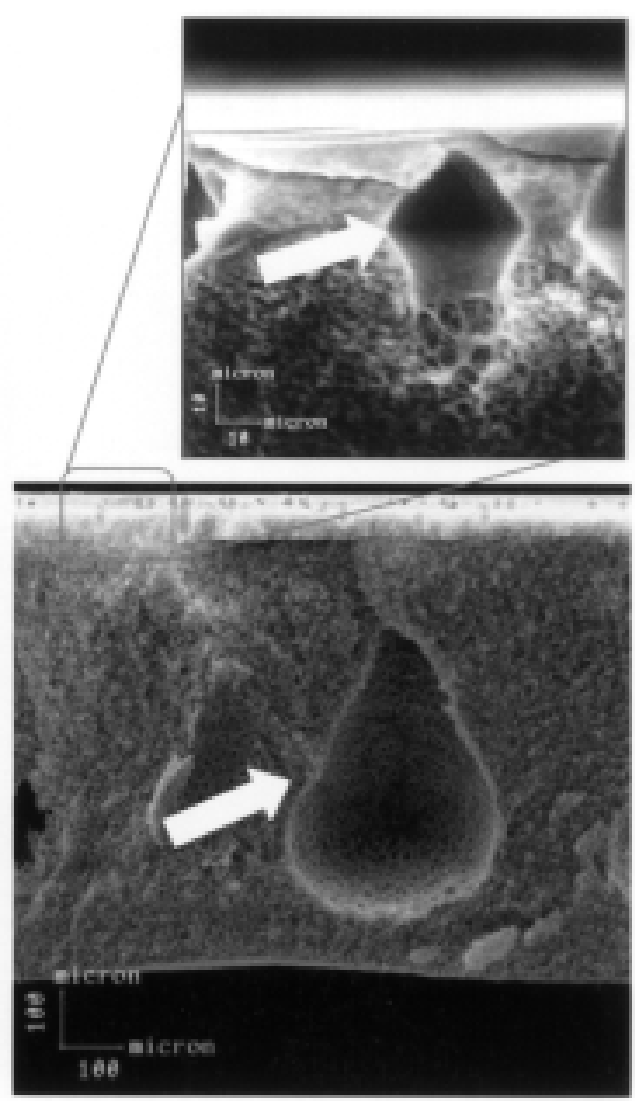

$30 \mathrm{~s}$

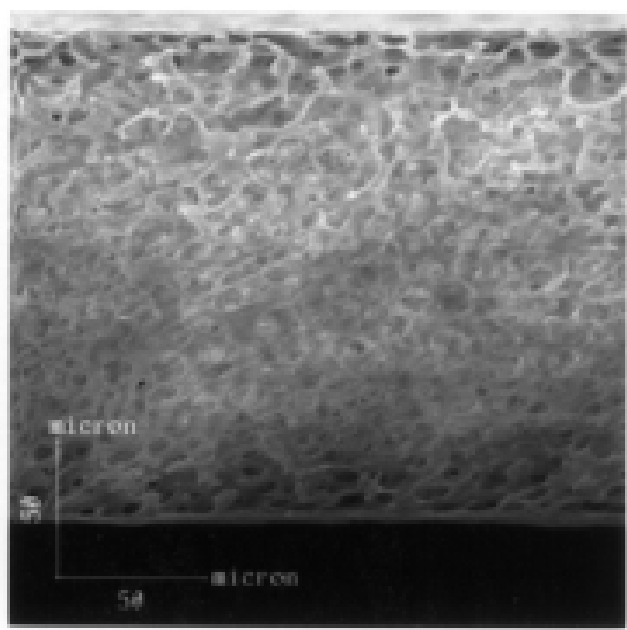

30 minutos

Figura 6. Fotomicrografias das seções transversais das membranas de camada dupla. 
um minuto e meio de acompanhamento da mesma, isto mostra que a precipitação da solução ainda não tinha sido iniciada neste intervalo. Por outro lado, após os 30 minutos de exposição ao ambiente, a solução se tornou turva. Esse fato sugere que a precipitação iniciou-se durante o tempo de exposição devido à evaporação do solvente volátil (S) e à absorção de água (NS) do ambiente. A precipitação total desta solução, no entanto, ocorreu após a sua imersão no banho de precipitação.

A Figura 6 mostra a influência do tempo de exposição do filme polimérico ao ambiente, antes de sua imersão no banho de precipitação (água destilada), sobre a morfologia das membranas de camada dupla.

A partir da Figura 6, observa-se que as membranas de camada dupla produzidas apresentaram total aderência entre as camadas formadas pelas duas soluções. Esta aderência pode estar atribuída às condições de transferência de massa entre as duas soluções poliméricas espalhadas simultaneamente, que atuam no sentido de retardar a precipitação nas regiões próximas à interface entre as mesmas, permitindo assim um tempo maior para interpenetração destas soluções, antes que ocorra o processo de separação de fases líquido-líquido.

Para as membranas com tempos de exposição 10 , 30 e 60s, observa-se uma estabilização de ambas as soluções poliméricas, dando origem a macrovazios (indicados por setas na Figura 6) nas duas camadas [macroporos formados pelo crescimento dos núcleos da fase pobre em polímero devido às condições favoráveis de precipitação com atraso ao longo do filme polimérico]. Esta estabilização pode ser justificada pela transferência de massa entre as duas soluções poliméricas (passagem de DMF da solução A em direção a solução B estabiliza a camada da pele, enquanto que, a camada porosa mantém-se estável pela difusão de acetona da solução B em direção a solução A). Na membrana com tempo de exposição de $60 \mathrm{~s}$, a combinação entre as concepções de formação das diferentes camadas, possibilitou que macrovazios, oriundos da camada superior, encontrassem condições de transferência de massa adequadas para continuarem a se desenvolver através da outra camada.

$\mathrm{Na}$ membrana exposta por 30 minutos ao ambiente, obtém-se uma morfologia com poros interconectados, a qual é característica de separação espinodal, freqüentemente observada em soluções contendo PVP. Sugere-se que esta morfologia seja decorrente da interpenetração das soluções e, consequiente presença do PVP na camada superior.
Cabe salientar que não foram observados poros visíveis na superfície superior das membranas, estando o tamanho dos mesmos fora dos limites de resolução alcançado pelo Microscópio Eletrônico de Varredura $(0,01 \mu \mathrm{m})$.

\section{Propriedades de Transporte das Membranas de Camada Dupla}

O fluxo de permeado e a rejeição ao soluto $(\mathrm{NaCl}$ para a OI e PEG para a NF) foram as propriedades de transporte empregadas para caracterização das membranas de camada dupla produzidas.

A Tabela 1 mostra o efeito do tempo de exposição do filme polimérico ao ambiente sobre as propriedades de transporte das membranas de camada dupla, em testes de permeação com solução aquosa de $\mathrm{NaCl}$ (testes de OI). A partir desta Tabela observa-se um efeito acentuado do tempo de exposição sobre as características de transporte das membranas resultantes, verificando-se um aumento no fluxo de permeado para os pequenos tempos de exposição (10, 30 e 60 segundos), sem mostrar grande variação na rejeição salina, e uma queda significativa do fluxo de permeado quando o filme foi exposto por 1800 segundos ao ambiente.

A tendência crescente do fluxo de permeado, para estes três primeiros tempos de exposição, está associada ao aumento da porosidade superficial das membranas. Uma possível hipótese para explicar este aumento é a presença de maior quantidade de PVP na camada superior das membranas com o aumento do tempo de exposição. Pode-se supor que a passagem de DMF da solução A para a solução B promove o arraste de PVP entre estas soluções. De fato, ao observar a seção transversal destas membranas, nota-se um aumento na porosidade das camadas próximas a sua superfície superior. Esse aumento no tamanho dos poros promove a diminuição da resistência ao transporte de solutos através da matriz polimérica, favorecendo o comportamen-

Tabela 1. Efeito do tempo de exposição sobre as propriedades de transporte das membranas de camada dupla em testes de permeação com solução aquosa de $\mathrm{NaCl}$ (testes de OI).

\begin{tabular}{ccc}
\hline $\begin{array}{c}\text { Tempo de } \\
\text { exposição } \\
(\mathbf{s})\end{array}$ & $\begin{array}{c}\text { Fluxo de permeado } \\
\text { (solução salina) } \\
{\left[\mathbf{L} / \mathbf{m}^{2} \cdot \mathbf{h}\right]}\end{array}$ & $\begin{array}{c}\text { Rejeição salina } \\
(\%)\end{array}$ \\
\hline 10 & 243 & 3 \\
30 & 394 & 2 \\
60 & 648 & 4 \\
1800 & 33 & 39 \\
\hline
\end{tabular}


to crescente do fluxo de permeado. No caso da membrana com tempo de exposição de 60s o prolongamento de macrovazios da camada superior para a inferior também promove uma diminuição na resistência ao transporte de massa através da membrana.

Como apresentado anteriormente, a membrana com tempo de exposição de 1800 segundos (30 minutos) possui morfologia porosa (Figura 6), decorrente da interpenetração entre as duas soluções e conseqüiente presença de PVP na sua camada superior. O menor fluxo de permeado e a maior rejeição salina, verificados para esta membrana, podem ser atribuídos aos efeitos de deformação mecânica ocorridos na seção transversal da mesma durante a sua compactação (Figura 7). Esses efeitos serão discutidos com mais detalhes na seção que mostra a influência do tratamento térmico nas características de transporte das membranas.

Com base nos resultados mostrados na Tabela 1 é possível observar baixos valores de rejeição salina, indicando que, provavelmente, a superfície superior das membranas duplas apresenta defeitos. Este fato pode ser atribuído à não uniformidade da pele seletiva causada pela absorção de água durante a exposição do filme ao ambiente. Com o objetivo de reduzir estes defeitos, as membranas planas de camada dupla foram submetidas ao tratamento térmico num banho isotérmico $\left(90^{\circ} \mathrm{C}\right)$ de água microfiltrada durante 1 hora.

Na Tabela 2 são apresentados os resultados das membranas de camada dupla com tratamento térmico, mostrando a influência do mesmo nas propriedades de transporte das membranas em testes de permeação com solução aquosa de $\mathrm{NaCl}$ (teste de $\mathrm{OI}$ ) e com solução aquosa de PEG (testes de NF). Nesta Tabela, observa-se que o tratamento térmico foi eficiente, aumentando os valores da rejeição salina de todas as membranas de camada dupla estudadas. Os valores de fluxo de permeado, com exceção da membrana exposta por 30 segundos ao ambiente, foram reduzidos. $\mathrm{O}$ alto valor de fluxo da membrana exposta por 30 segundos ao ambiente, e o resultado de maior rejeição salina do que rejeição a PEG (molécula maior do que o $\mathrm{NaCl}$ ), são indicativos de defeitos ou fissuras na mesma. $\mathrm{O}$ aumento da seletividade das membranas e a redução da permeabilidade das mesmas com o tratamento térmico ocorrem devido ao aumento da mobilidade segmental do polímero, levando à uma contração da membrana tratada pela redução do volume livre da matriz polimérica ${ }^{[10]}$.

A membrana com tempo de exposição de 10 segundos apresentou elevado valor de rejeição do PEG $-82 \%$, com fluxos de permeado relativamente alto $-42 \mathrm{~L} / \mathrm{m}^{2}$.h, mostrando-se viável a aplicação desta membrana em processos industriais.

No presente trabalho, dentre todas as condições investigadas no preparo de membranas planas duplas, a membrana com tempo de exposição de 1800 segundos foi a que apresentou a maior rejeição salina $(\mathrm{NaCl})-63 \%$ e o menor fluxo de permeado $-7 \mathrm{~L} / \mathrm{m}^{2} . h$. Como a análise morfológica desta membrana indicou elevada porosidade na sua seção transversal (Figura 6), pode-se atribuir as melhores características de transporte aos efeitos de deformação mecânica ocorridos na matriz polimérica durante a sua etapa de compactação. A alta pressão aplicada ( $\mathrm{P}=28$ bar) transversalmente na superfície superior da membrana provoca uma drástica redução irreversível na sua espessura (na ordem de 60\%, Figura 7). Esta redução pode levar a uma densificação da membrana, mantendo caminhos intersticiais ao longo de sua seção transversal. Desta forma, após a compactação, os valores de rejeição e de fluxo seriam determinados pela soma das duas parcelas: uma devido à difusão através da parte densa da matriz polimérica e

Tabela 2. Efeito do tratamento térmico sobre as propriedades de transporte das membranas de camada dupla em testes de permeação com soluções aquosa de $\mathrm{NaCl}$ e de PEG.

\begin{tabular}{|c|c|c|c|c|}
\hline \multirow{2}{*}{$\begin{array}{c}\text { Tempo de exposição } \\
\text { (s) }\end{array}$} & \multicolumn{2}{|c|}{ Fluxo de permeado*** } & \multirow{2}{*}{$\begin{array}{c}\text { Rejeição Salina } \\
(\%)\end{array}$} & \multirow{2}{*}{$\begin{array}{c}\text { Rejeição PEG } \\
(\%)\end{array}$} \\
\hline & Sol.* Salina & Sol.* PEG & & \\
\hline 10 & 66 & 42 & 27 & 82 \\
\hline 30 & 465 & 464 & 10 & 6 \\
\hline 60 & 91 & 61 & 24 & 61 \\
\hline 1800 & 7 & 8 & 63 & 53 \\
\hline
\end{tabular}

\footnotetext{
* solução
}

$* *\left[\mathrm{~L} / \mathrm{m}^{2} . \mathrm{h}\right]$ 


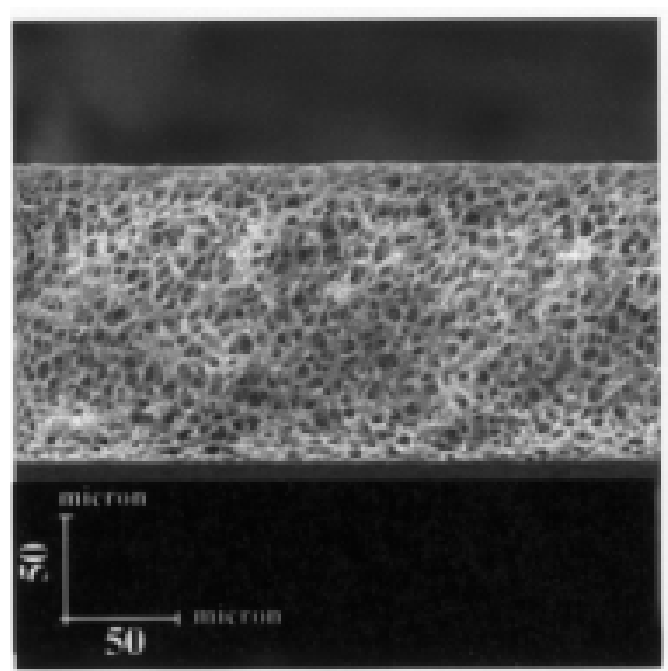

(a)

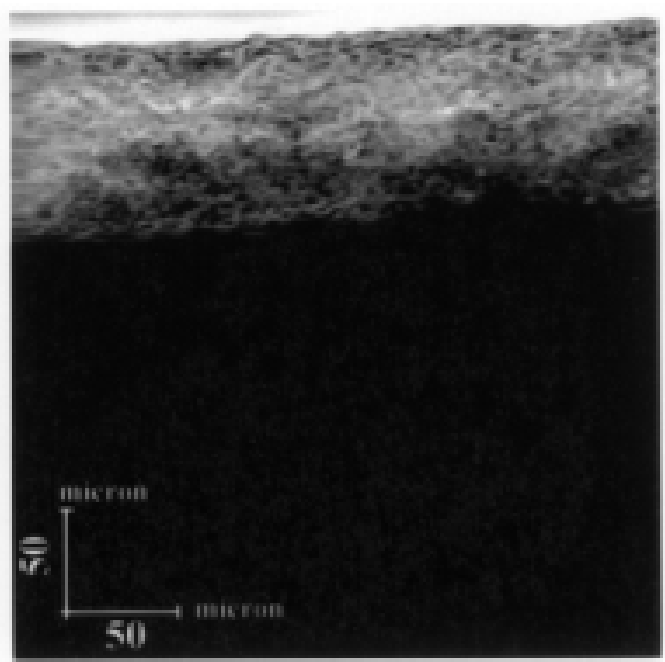

(b)

Figura 7. Efeitos da etapa de compactação da membrana plana dupla com exposição prolongada ao ambiente (30 minutos) sobre a sua morfologia: (a) antes da compactação e (b) após a compactação.

a outra devido ao escoamento nos interstícios resultantes da compactação (contribuição convectiva). Logo, o baixo valor do fluxo de permeado pode ser justificado pela presença da contribuição difusiva, enquanto o valor intermediário da rejeição salina (63\%) pode estar associado à menor rejeição do $\mathrm{NaCl}$ na parcela convectiva $^{[8]}$.

Comparando as características de transporte desta membrana para os diferentes solutos - $\mathrm{NaCl}$ e PEG (Tabela 2), observa-se um comportamento não esperado, visto que a rejeição salina (molécula menor) foi maior do que a rejeição ao PEG. A parte densa da matriz polimérica rejeita ambos os solutos. A alimentação do sistema com solução aquosa de $\mathrm{NaCl}$ (composto iônico) pode favorecer a ocorrência de efeitos eletrocinéticos durante a etapa de caracterização, promovida devido à presença de cargas elétricas ao longo da seção transversal da membrana. Estes efeitos levam a uma exclusão dos íons $\mathrm{Na}^{+}$e $\mathrm{Cl}^{-}$no escoamento da solução pelos interstícios resultantes da etapa de compactação, aumentando a contribuição da parte convectiva nas características de transporte da membrana. Este fato justifica o comportamento observado acima, pois na caracterização com PEG estes efeitos eletrocinéticos são reduzidos e a rejeição nos interstícios tende a zero ${ }^{[8]}$.

\section{Conclusões}

Com base nos resultados obtidos, podemos concluir que:
- É possível a obtenção de membranas anisotrópicas compostas, através da técnica de espalhamento duplo, em uma única etapa, com total aderência das camadas formadas pelas soluções. Esta aderência foi atribuída às condições de transferência de massa, as quais retardam a precipitação na região próxima à interface entre as soluções.

- Os resultados da velocidade de precipitação e de morfologia das membranas de camada dupla, mostraram-se concordantes entre si, indicando uma estabilização de ambas as soluções poliméricas, sendo que as membranas apresentaram macrovazios nas duas camadas.

- O tratamento térmico mostrou-se eficiente, promovendo grande aumento da seletividade das diferentes membranas produzidas, tornando-as adequadas aos processos de NF e OI. As membranas desenvolvidas apresentaram rejeição salina $(\mathrm{NaCl})$ entre 24 $63 \%$ e rejeição molecular (PEG), entre $53-82 \%$, com fluxos de permeados relativamente altos, mostrando o potencial de aplicação destas membranas.

\section{Agradecimentos}

Roberto B. de Carvalho agradece à CAPES (Ministério da Educação) pela bolsa de Mestrado concedida.

\section{Referências bibliográficas}

1. Mulder, M. - "Basic Principles of Membrane Technology”, Kluwer Academic Publishers, Boston (1991). 
2. Kesting, R. E. - "Synthetic Polymeric Membranes", Mcgraw Hill, New York (1972).

3. Baker, R. W.; Cussler, E. L; Eykamp, W.; Koros, W. J.; Riley, R. L \& Strathmann, H. - "Membrane separation systems. Recent development and future directions", Noyes Data Co., New Jersey (1991).

4. Loeb, S. \& Sourirajam, S. - "Sea water desmineralization by means of an osmotic membrane", Adv. Chem. Ser., 28, p.117 (1962).

5. Pereira, C. C. - "Desenvolvimento de Fibras Ocas Anisotrópicas para Separação de Gases", Tese de Doutorado, Programa de Engenharia Química da COPPE, Universidade Federal do Rio de Janeiro, Brasil (1999).

6. Kools, W. F. C. - "Membrane formation by phase inversion in multicomponent polymer systems: mechanisms and morphologies", Ph.D. dissertation, Twente University, The Netherlands (1998).
7. Meuleman, E. - "Removal of trace organic contaminants from water by pervaporation", Ph.D. dissertation, Twente University, The Netherlands (1997).

8. Carvalho, R. B. de - "Membranas Anisotrópicas para Nanofiltração e Osmose Inversa Preparadas pelo Processamento Simultâneo de Duas Soluções Poliméricas", Tese de Mestrado, Programa de Engenharia Química da COPPE, Universidade Federal do Rio de Janeiro, Brasil (2001).

9. Egler, M. A. G.; "Fracionamento de Misturas Ternárias Glicose/Etanol/Água por Osmose Inversa com Membranas de Acetato de Celulose", Tese de Mestrado, Programa de Engenharia Química da COPPE, Universidade Federal do Rio de Janeiro, Brasil (1984).

10. Lonsdale, H. K. \& Podall, H. E. - "Reverse Osmosis Membrane Research”, Plenum Press, New York (1972).

Recebido: 01/02/01

Aprovado: 20/07/01 